\title{
Existencia de solución y su comportamiento respecto a un parámetro para un modelo de ondas en un fluido viscoso
}

\author{
Luis Milla Garcia ${ }^{1}$ y Yolanda Santiago Ayala ${ }^{2}$
}

Resumen: En este trabajo estudiamos la existencia, unicidad y dependencia continua de la solución de la ecuación lineal homogénea KdV-Kuramoto-Sivashinsky en espacios de Sobolev periódicos. Realizamos esto usando la teoría de semigrupos y la teoría de Fourier en distribuciones periódicas. También, usando las inmersiones entre los espacios de Sobolev obtenemos propiedades adicionales de regularidad. Además, probamos algunas afirmaciones hechas en [8]. Finalmente, analizamos el comportamiento de la solución respecto a un parámetro, probando que su límite es la solución de un problema de Cauchy cuyo semigrupo asociado es la restricción de un grupo.

Palabras clave: existencia de solución; ecuación KdV-Kuramoto-Sivashinski; espacios de Sobolev periódico; semigrupos.

\section{Existence of solution and its behavior with respect to one parameter for a wave model in a viscous fluid}

\begin{abstract}
In this work we study the existence, uniqueness and continuous dependence of the solution of the KdV-Kuramoto-Sivashinsky homogeneous linear equation in periodic Sobolev spaces. We do this using semigroup theory and Fourier theory on periodic distributions. Also, using the immersions between the Sobolev spaces we obtain regularity additional properties. Furthermore, we proved some claims done in [8].Finally, we analyze the behavior of the solution with respect to one parameter, proving that its limit is the solution of a Cauchy problem whose associated semigroup is the restriction of a group.
\end{abstract}

Keywords: existence of solution; KdV-Kuramoto-Sivashinski equation; periodic Sobolev spaces; semigroups.

Recibido: 27/02/2020. Aceptado: 25/07/2020. Publicado online: 18/08/2020.

(C) Los autores. Este artículo es publicado por la Revista PESQUIMAT de la Facultad de Ciencias Matemáticas, Universidad Nacional Mayor de San Marcos. Este es un artículo de acceso abierto, distribuido bajo los términos de la licencia Creative Commons Atribucion-No Comercia-CompartirIgual 4.0 Internacional.(http://creativecommons.org/licenses/by-nc-sa/4.0/) que permite el uso no comercial, distribución y reproducción en cualquier medio, siempre que la obra original sea debidamente citada. Para uso comercial, por favor póngase en contacto con revistapesquimat.matematica@unmsm.edu.pe

\footnotetext{
${ }^{1}$ UNMSM, Facultad de Ciencias Matemáticas. e-mail: lmillag@unmsm.edu.pe

${ }^{2}$ UNMSM, Facultad de Ciencias Matemáticas, e-mail:ysantiagoa@unmsm.edu.pe
} 


\section{Introducción}

Las ecuaciones diferenciales parciales de evolución tienen gran importancia dentro de la matemática actual debido al papel que juegan dentro de la formulación de modelos para describir fenómenos dinámicos de las ciencias físicas y naturales.

Uno de los aspectos de mayor interés en el estudio de las ecuaciones de evolución es lo relacionado con la existencia, unicidad, regularidad y dependencia continua respecto al dato inicial de la solución del problema de Cauchy o problema de valores iniciales (PVI) asociado a la ecuación de evolución.

Empezamos recordando que la ecuación de Kuramoto-Sivashinsky (K-S):

$$
u_{t}+u u_{x}+u_{x x}+u_{x x x x}=0
$$

data de mediados de 1970. La primera derivación fue hecha por Kuramoto en el estudio de ecuaciones de reacción-difusión modelando la reacción Belonsov-Zabotinski. Dicha ecuación fue también desarrollada por Sivashinsky en dimensiones más altas en láminas temperadas frontales. Por otro lado, la bien conocida, ecuación no lineal de KdV

$$
u_{t}+u u_{x}+u_{x x x}=0
$$

así como la K-S fueron estudiadas por muchos autores, podemos citar por ejemplo Bona J.L., en [1]. Kato T., en [3] y otros. Del acoplamiento de los dos modelos se deduce el modelo para ondas en un fluido viscoso KdV-Kuramoto-Sivashinsky:

$$
u_{t}+u_{x x x}+\beta\left(u_{x x x x}+u_{x x}\right)=0 \text { en } H_{p e r}^{s-4} \text { con } u(0)=\phi \in H_{p e r}^{s}
$$

considerando $\beta$ una constante positiva, $s$ un número real y $H_{p e r}^{s}$ denotando por al espacio de Sobolev periódico de orden $s$. Las derivadas de tercer y cuarto orden son los términos dispersivos y disipativos respectivamente deducidos en [10].

Físicamente es un modelo que describe, en una dimensión espacial, la propagación de ondas en medios viscosos. Nos preguntamos si el modelo posee solución, y si existe, ¿es única?. En efecto, se consigue probar la existencia y unicidad de solución del PVI $(P)$, y además, que la solución depende continuamente respecto al dato inicial. Además analizamos el comportamiento de la solución del problema de Cauchy a un parámetro probándose que su límite es la solución de un problema Cauchy cuyo semigrupo asociado es la restricción de un grupo.

Algunos trabajos de la existencia vía semigrupos de operadores lineales fueron desarrollados por Liu, Z. and Zheng, S. [5], Muñoz Rivera [6], Pazy, A. [7], Yolanda Santiago [8], [9].

El presente trabajo, en su totalidad, esta constituido por secciones que describiremos a continuación:

La sección 2 recoge algunos resultados conocidos de la teoría de los espacios de Sobolev periódico y teoría de operadores lineales que serán necesarios a lo largo del trabajo. En la sección 3 se indica los teoremas del buen planteamiento global y la regularidad del modelo KdV-K-S de manera intuitiva. En la sección 4 mostramos una versión más fina de los teoremas de la sección 3 usando la teoría de semigrupos de operadores. En la sección 5 se estudia el comportamiento en el limite de la familia de soluciones $\left\{u_{\beta}\right\}_{\beta>0}$. En la sección 6 damos una versión mas fina de la sección 5 vía la teoría de grupos de operadores. Finalmente en la sección 7 damos las conclusiones de nuestro estudio. 


\section{Preliminares}

El objetivo de esta sección es recoger algunos resultados conocidos acerca de los espacios de Sobolev periódicos, Teoría de Operadores lineales y Teoría de Semigrupos, que se usaron en el trabajo.

\subsection{Espacio de Sobolev Periódico}

Para la prueba de los siguientes resultados, citamos [2] y [9].

Definición 1. Sea $s \in \mathbb{R}$. El espacio de Sobolev $H_{\text {per }}^{s}=H_{\text {per }}^{s}([-\pi, \pi])$ es el conjunto de todos los $f \in \mathcal{P}^{\prime}$ tal que:

$$
\|f\|_{s}^{2}=2 \pi \sum_{k=-\infty}^{\infty}\left(1+|k|^{2}\right)^{s}|\widehat{f}(k)|^{2}<\infty
$$

$\mathcal{P}$ es el espacio de las funciones periódicas infinitamente diferenciables de periodo $2 \pi ; \mathcal{P}^{\prime}$ es el dual topológico de $\mathcal{P}$, conocido como el espacio de las distribuciones periódicas de periodo $2 \pi$. En otras palabras, una distribución $f$ esta en el espacio de Sobolev $H_{\text {per }}^{s}$ si y sólo si: $\left(\left(1+|k|^{2}\right)^{s / 2} \widehat{f}(k)\right)_{k \in \mathbb{Z}} \in l^{2}=l^{2}(\mathbb{Z})$. Denotemos por $l_{s}^{2}=l_{s}^{2}(\mathbb{Z})$ el espacio de todas las sucesiones $\alpha=\left(\alpha_{k}\right)_{k \in \mathbb{Z}}$ con

$$
\|\alpha\|_{l_{s}^{2}}=\left[2 \pi \sum_{k=-\infty}^{\infty}\left(1+|k|^{2}\right)^{s}\left|\alpha_{k}\right|^{2}\right]^{1 / 2} .
$$

Así $f \in H_{\text {per }}^{s}$ si y sólo si $(\widehat{f}(k))_{k \in \mathbb{Z}} \in l_{s}^{2}$; en este caso, $\|f\|_{s}=\|\widehat{f}\|_{l_{s}^{2}}$. Es fácil ver que para todo $s \in \mathbb{R}, H_{\text {per }}^{s}$ es un espacio de Hilbert, con respecto al producto interno:

$$
\langle f \mid g\rangle_{s}=2 \pi \sum_{k=-\infty}^{\infty}\left(1+|k|^{2}\right)^{s} \widehat{f}(k) \overline{\widehat{g}(k)}
$$

En el caso $s=0$, obtenemos el espacio de Hilbert que es isométricamente isomorfo a: $L^{2}([-\pi, \pi])$, el conocido espacio de clases de equivalencia de funciones cuadrado integrables en el sentido de Lebesgue. En lo que sigue $H_{p e r}^{0}$ a menudo se denotará por $L_{p e r}^{2}$.

Proposición 1. Sea: $s, r$ tal que: $s \geq r$, entonces: $H_{p e r}^{s} \hookrightarrow H_{p e r}^{r}$, i.e. $H_{\text {per }}^{s}$ está inmerso continuamente $y$ densamente en $H_{\text {per }}^{r} y$

$$
\|f\|_{r} \leq\|f\|_{s}, \forall f \in H_{p e r}^{s} .
$$

En particular, tenemos que si $s \geq 0$, entonces:

$$
H_{p e r}^{s} \subset L^{2}([-\pi, \pi]) .
$$

Además, vale la identificación isométricamente isomorfo

$$
\left(H_{\text {per }}^{s}\right)^{\prime} \equiv H_{\text {per }}^{-s}, \quad \forall s \in \mathbb{R},
$$

donde la dualidad es implementada por el par:

$$
<f, g>_{s}=2 \pi \sum_{k=-\infty}^{+\infty} \hat{f}(k) \hat{g}(k), \forall f \in H_{p e r}^{-s}, g \in H_{p e r}^{s} .
$$


Teorema 1. Si $s>\frac{1}{2}$ entonces se verifican.

1. La serie de Fourier de $f \in H_{\text {per }}^{s}$ converge absoluta y uniformemente en $[-\pi, \pi]$, i.e., la serie de Fourier de $f$

$$
\sum_{k=-\infty}^{+\infty} \hat{f}(k) e^{i k x}
$$

converge absolutamente y uniformemente en $[-\pi, \pi]$.

2. Lema de inmersión de Sobolev: $H_{\text {per }}^{s} \hookrightarrow C_{\text {per }}$ y hace corresponder a $f \in H_{\text {per }}^{s}$ la función $g \in C_{\text {per }}$, donde $g(x)=\sum_{k=-\infty}^{\infty} \widehat{f}(k) e^{i k x}$ y satisface

$$
\|g\|_{\infty} \leq\|f\|_{l^{1}} \leq C\|f\|_{s}, \forall f \in H_{p e r}^{s} .
$$

\subsection{Familias de operadores acotados.}

Daremos las nociones de semigrupos y grupos unitarios de operadores sobre espacios de Banach. Estos operadores son muy útiles al resolver el problema de Cauchy (PVI) abstracto de tipo periódico. Una descripción mas detallada de algunos contenidos de esta sección puede consultarse en [5], [6], [7].

Definición 2. Sea $X$ un espacio de Banach. Un semigrupo paramétrico fuertemente continuo en $X$ (o simplemente un semigrupo de clase $C_{0}$ ) es una aplicación $S:[0, \infty) \rightarrow \mathcal{L}(X)$ tal que.

1. $S(0)=I$ donde $I$ es el operador identidad en $\mathcal{L}(X)$,

2. $S(t+r)=S(t) S(r), \forall t, r \in(0, \infty)$,

3. $\lim _{t \rightarrow 0^{+}}\|S(t) \phi-\phi\|=0, \forall \phi \in X$

y será denotado por: $\{S(t)\}_{t \geq 0}$.

Observación 1. Si $\{S(t)\}_{t \geq 0}$ es un semigrupo de clase $C_{0}$ entonces satisface:

$$
\lim _{t \rightarrow r}\|S(t) \phi-S(r) \phi\|=0, \forall r \in[0, \infty), \forall \phi \in X,
$$

es decir, la aplicación de $[0, \infty)$ a $X$ enviando $t$ a $S(t) \phi$ es continuo.

Definición 3. Sea $\{S(t)\}_{t \geq 0}$ un semigrupo de clase $C_{0}$, si satisface:

$$
\|S(t)\|_{\mathcal{L}(X)} \leq 1, \forall t \in[0, \infty)
$$

entonces diremos que el semigrupo de clase $C_{0}$ es de contracción.

Definición 4. Sea $H_{j}, j=1,2$ espacios de Hilbert. Un operador $T \in \mathcal{B}\left(H_{1}, H_{2}\right)$ es una isometría si $\|T h\|_{H_{1}}=\|h\|_{H_{2}}$ para todo $h \in H_{1}$ (en particular $T$ es inyectiva). $T$ es unitario si es una isometría sobreyectiva.

Definición 5. Sea $H$ un espacio de Hilbert. Un grupo unitario uniparamétrico fuertemente continuo en $H$ es un mapeo $t \in \mathbb{R} \rightarrow T(t) \in \mathcal{B}(H)$ tal que:

(a) $T(t)$ es unitario para todo $t \in \mathbb{R}$,

(b) $T\left(t+t^{\prime}\right)=T(t) T\left(t^{\prime}\right), \quad \forall t, t^{\prime} \in \mathbb{R}$, 
(c) $\quad \lim _{t \rightarrow t^{\prime}}\left\|T(t) \phi-T\left(t^{\prime}\right) \phi\right\|_{H}=0, \quad \forall t \in \mathbb{R}$.

Observación 2. De (b) deducimos que $T(0)=I$.

En efecto, haciendo $t^{\prime}=0$ en (b) tenemos: $T(t)=T(t) T(0)$. Luego $T(t)\{I-T(0)\}=0$, esto es, $I=T(0)$.

La observación 2, nos dice que un grupo unitario uniparamétrico fuertemente continuo es en particular un $C_{0}$ semigrupo.

\section{El problema de Cauchy de la ecuación KdV-K-S}

En esta sección obtenemos vía inmersión de Espacios de Sobolev algunas desigualdades adicionales comprendidas en el corolario 1 del artículo [8]. Tenemos.

Teorema 2. Sea s un número real fijo, $\beta>0$ y el problema:

$$
(P) \mid \begin{aligned}
& u \in C\left([0,+\infty\rangle, H_{\text {per }}^{s}\right) \\
& \partial_{t} u+\partial_{x}^{3} u+\beta\left(\partial_{x}^{4} u+\partial_{x}^{2} u\right)=0 \in H_{p e r}^{s-4} \\
& u(0)=\phi \in H_{\text {per }}^{s} .
\end{aligned}
$$

Entonces $(P)$ está globalmente bien puesto, esto es, $\exists ! u \in C\left([0, \infty), H_{\text {per }}^{s}\right)$ satisfaciendo la ecuación $(P)$ y la aplicación $: \phi \rightarrow u$, que a cada condición inicial $\phi$ le asigna la solución u del PVI $(P)$ es continua. Además, dicha solución satisface la regularidad:

$$
u(t) \in H^{\infty}, \forall t>0
$$

con $\|u(t)\|_{r} \leq C\|\phi\|_{s}, \forall r \in \mathbb{R} \quad y \quad t>0$, donde

$$
H^{\infty}:=\bigcap_{r \in \mathbb{R}} H_{p e r}^{r} .
$$

Ahora como consecuencia del teorema 2 obtenemos los siguientes resultados.

Corolario 1. Con las hipótesis del precedente teorema obtenemos:

$$
u \in C\left([0, \infty), H_{p e r}^{s}\right), \forall r \leq s .
$$

También se satisface

$$
\|u(t)\|_{r} \leq\|\phi\|_{s}, \forall t \geq 0, \forall r \leq s \quad y \quad \underset{t \geq 0}{\sup \|u(t)\|_{r} \leq\|\phi\|_{s}, \forall r \leq s}
$$

Si $r>s$ entonces $\|u(t)\|_{r} \leq \sqrt{C^{*}}\|\phi\|_{s}, \forall t>0$ donde $C^{*}$ es tal que $|G(k, t)| \leq C^{*}, \forall k \in \mathbb{Z}$, $\forall t>0$, con $G(k, t)=e^{2 \beta\left(k^{2}-1\right) k^{2} t}\left(1+k^{2}\right)^{r-s}$.

Finalmente

$$
\|u(t)\|_{r} \leq \min \left\{1, \sqrt{C^{*}}\right\}\|\phi\|_{s}, \forall t>0, \forall r \in \mathbb{R} .
$$

Corolario 2. La única solución de la ecuación $(P)$ es:

$$
u(x, t)=\sum_{k=-\infty}^{+\infty} e^{i k^{3} t-\beta\left(k^{2}-1\right) k^{2} t} \widehat{\phi}(k) e^{i k x} .
$$




\section{Enfoque vía Semigrupos}

En esta sección presentamos una familia de operadores que forman un semigrupo de clase $C_{0}$, como lo hacemos en el teorema 3. Finalmente, establecemos el teorema 4, que es una versión fina del teorema 2, basada en el semigrupo $\{S(t)\}_{t \geq 0}$ introducido.

Teorema 3. Sea $\beta>0$ y $s \in \mathbb{R}$. La aplicación $S:[0, \infty) \rightarrow \mathcal{L}\left(H_{\text {per }}^{s}\right)$, que asigna a $t$ el operador $S(t)=e^{-\left(\partial_{x}^{3}+\beta\left(\partial_{x}^{4}+\partial_{x}^{2}\right)\right) t}$, es decir, aplica $S(t) \phi=\left\{e^{\left(i k^{3} t-\beta\left(k^{4}-k^{2}\right)\right) t} \widehat{\phi}(k)\right\}^{\vee},\{S(t)\}_{t \geq 0}$ es un semigrupo de clase $C_{0}$ de contracción en $H_{p e r}^{s}$. Además las siguientes afirmaciones se cumplen:

1. Si $\phi \in H_{p e r}^{s}$ entonces $S(\cdot) \phi \in C\left([0, \infty), H_{p e r}^{s}\right)$.

2. La aplicación $\phi \rightarrow S(\cdot) \phi$ es continua y verifica:

$$
\begin{aligned}
\left\|S(t) \phi_{1}-S(t) \phi_{2}\right\|_{s} & \leq\left\|\phi_{1}-\phi_{2}\right\|_{s}, \forall t>0, \\
\sup _{t>0}\left\|S(t) \phi_{1}-S(t) \phi_{2}\right\|_{s} & \leq\left\|\phi_{1}-\phi_{2}\right\|_{s} .
\end{aligned}
$$

Demostración. La prueba lo hemos organizado de la siguiente forma.

1. Primero observemos que

$$
S(0) \phi=\left\{e^{\left(i k^{3}(0)-\beta\left(k^{4}-k^{2}\right)\right)(0)} \widehat{\phi}(k)\right\}^{\vee}=\{\widehat{\phi}(k)\}^{\vee}=\phi
$$

así $S(0)=I$. Además de la propiedad de linealidad de la transformada de Fourier y de su inversa, tenemos que $S(t)$ es lineal, es decir, $S(t) \in \mathcal{L}\left(H_{\text {per }}^{s}\right)$.

2. Si $\phi \in H_{\text {per }}^{s}$ probaremos que $S(t) \phi \in H_{\text {per }}^{s} \quad$ y $\|S(t) \phi\|_{s} \leq\|\phi\|_{s}$, esto es, $\|S(t)\|<1$.

En efecto, se tiene:

$$
\begin{aligned}
\|S(t) \phi\|_{s}^{2} & =2 \pi \sum_{k=-\infty}^{+\infty}\left(1+|k|^{2}\right)^{s}\left|e^{i k^{3} t-\beta\left(k^{2}-1\right) k^{2} t} \widehat{\phi}(k)\right|^{2} \\
& =\left.2 \pi \sum_{k=-\infty}^{+\infty}\left(1+|k|^{2}\right)^{s}\left|e^{2 i k^{3} t}\right|\left|e^{-2 \beta\left(k^{2}-1\right) k^{2} t \mid}\right| \widehat{\phi}(k)\right|^{2} \\
& =2 \pi \sum_{k=-\infty}^{+\infty}\left(1+|k|^{2}\right)^{s}|\widehat{\phi}(k)|^{2} e^{-2 \beta\left(k^{2}-1\right) k^{2} t} \\
& \leq 2 \pi \sum_{k=-\infty}^{+\infty}\left(1+|k|^{2}\right)^{s}|\widehat{\phi}(k)|^{2}=\|\phi\|_{s}^{2}<\infty
\end{aligned}
$$

Entonces:

$S(t) \phi \in H_{\text {per }}^{s} \mathrm{y}\|S(t) \phi\|_{s} \leq\|\phi\|_{s}$, esto es, $S(t) \in \mathcal{L}\left(H_{\text {per }}^{s}\right)$ con $\|S(t)\| \leq 1$.

3. Seguidamente probaremos que: $S(t+r)=S(t) S(r), \forall t, r \geq 0$.

$$
\begin{aligned}
S(t+r) f(x) & =2 \pi \sum_{k=-\infty}^{+\infty} e^{i k^{3}(t+r)-\beta\left(k^{2}-1\right) k^{2}(t+r)} \widehat{f}(k) e^{i k x} \\
& =2 \pi \sum_{k=-\infty}^{+\infty} e^{i k^{3} t-\beta\left(k^{2}-1\right) k^{2} t}\left\{e^{i k^{3} r-\beta\left(k^{2}-1\right) k^{2} r} \widehat{f}(k)\right\} e^{i k x} \\
& =S(t) g(x),
\end{aligned}
$$


donde $g$ es tal que: $\widehat{g}(k)=e^{i k^{3} r-\beta\left(k^{2}-1\right) k^{2} r} \widehat{f}(k), \forall k \in \mathbb{Z}$. Así

$$
g(x)=2 \pi \sum_{k=-\infty}^{+\infty} e^{i k^{3} r-\beta\left(k^{2}-1\right) k^{2} r} \widehat{f}(k) e^{i k x}=S(r) f(x)
$$

en consecuencia $S(t+r) f=S(t) S(r) f, \forall t, r \geq 0$.

4. Ahora, probaremos la continuidad de: $t \rightarrow S(t) \phi$

$$
\|S(t+h) \phi-S(t) \phi\|_{s} \rightarrow 0, \text { cuando, } h \rightarrow 0 .
$$

En efecto:

$$
\begin{aligned}
& \|S(t+h) \phi-S(t) \phi\|_{s}^{2} \\
& =2 \pi \sum_{k=-\infty}^{+\infty}\left(1+k^{2}\right)^{s}\left|\left(e^{i k^{3}(t+h)-\beta\left(k^{2}-1\right) k^{2}(t+h)}-e^{i k^{3} t} e^{-\beta\left(k^{2}-1\right) k^{2} t}\right) \widehat{\phi}(k)\right|^{2} \\
& =2 \pi \sum_{k=-\infty}^{+\infty}\left(1+k^{2}\right)^{s}|\widehat{\phi}(k)|^{2}\left|e^{2\left(i k^{3}-\beta\left(k^{4}-k^{2}\right)\right) t}\right| \mid\left(e^{\left(i k^{3}-\beta\left(k^{4}-k^{2}\right)\right) h}-\left.1\right|^{2}\right. \\
& =2 \pi \sum_{k=-\infty}^{+\infty}\left(1+k^{2}\right)^{s}|\widehat{\phi}(k)|^{2} e^{-2 \beta\left(k^{4}-k^{2}\right) t} \mid\left(e^{\left(i k^{3}-\beta\left(k^{4}-k^{2}\right)\right) h}-\left.1\right|^{2}\right. \\
& =2 \pi \sum_{k=-\infty}^{+\infty} \underbrace{\left(1+k^{2}\right)^{s}|\widehat{\phi}(k)|^{2} e^{-2 \beta\left(k^{4}-k^{2}\right) t}|M(k, h)|^{2}}_{I_{k, t, h}:=}
\end{aligned}
$$

donde

$$
H(k, h):=e^{\left(i k^{3}-\beta\left(k^{4}-k^{2}\right)\right) h}-1 .
$$

Observamos que $\lim _{h \rightarrow 0} H(k, h)=0$. Ahora necesitamos otra vez la convergencia uniforme de la serie para el intercambio de límites. Para esto, tomemos el término $I_{k, h, t}$ de la serie (5) y lo acotamos por una serie convergente, esto es:

$$
\begin{aligned}
I_{k, t, h} & =2 \pi\left(1+k^{2}\right)^{s}|\widehat{\phi}(k)|^{2}\left|e^{\left(i k^{3}-\beta\left(k^{2}-1\right) k^{2}\right)(t+h)}-e^{\left(i k^{3}-\beta\left(k^{2}-1\right) k^{2}\right) t}\right|^{2} \\
& \leq 2 \pi\left(1+k^{2}\right)^{s}|\widehat{\phi}(k)|^{2}(2)^{2}=8 \pi\left(1+k^{2}\right)^{s}|\widehat{\phi}(k)|^{2},
\end{aligned}
$$

donde hemos usado la desigualdad triangular (propiedad de la norma) y la desigualdad $e^{-\theta} \leq 1$ para $\theta \geq 0$. Así

$$
\sum_{k=-\infty}^{\infty} I_{k, t, h} \leq 4\|\phi\|_{s}^{2}<\infty
$$

entonces usando el M-test de Weierstrass [4, pag. 192] obtenemos que la serie (5) converge absoluta y uniformemente a una función continua en $h$ luego podemos intercambiar los límites, esto es:

$$
\lim _{h \rightarrow 0}\|S(t+h) \phi-S(t) \phi\|_{s}^{2}=\sum_{k=-\infty}^{\infty} \lim _{h \rightarrow 0} I_{k, h, t}=0
$$

y de esto concluimos (4):

$$
\lim _{h \rightarrow 0}\|S(t+h) \phi-S(t) \phi\|_{s}=0 .
$$


Observación 3. Tenemos que

$$
\lim _{t \rightarrow 0^{+}}\|S(t) \phi-\phi\|_{s}=0
$$

Observación 4. De la observación anterior 3 tenemos que: $\{S(t)\}_{t \geq 0}$ es un semigrupo de clase $C_{0}$. Así, por la observación 3 y la definición 3 , de la sección de preliminares, se tiene que $\{S(t)\}_{t \geq 0}$ es un semigrupo de contracción de clase $C_{0}$ en $H_{p e r}^{s}$. Sean $\phi_{1}$ y $\phi_{2}$ datos próximos en $H_{p e r}^{s}$, entonces se prueba que sus correspondientes soluciones $S(\cdot) \phi_{1}$ y $S(\cdot) \phi_{2}$ son próximos.

En efecto, desde que $\{S(t)\}_{t \geq 0}$ es de contracción para $t \geq 0$, tenemos:

$$
\left\|S(t) \phi_{1}-S(t) \phi_{2}\right\|_{s}=\left\|S(t)\left(\phi_{1}-\phi_{2}\right)\right\|_{s} \leq\left\|\phi_{1}-\phi_{2}\right\|_{s} .
$$

Tomando supremo sobre $(0, \infty)$ obtenemos:

$$
\sup _{t \in(0,+\infty)}\left\|S(t) \phi_{1}-S(t) \phi_{2}\right\|_{s} \leq\left\|\phi_{1}-\phi_{2}\right\|_{s} .
$$

De aquí tenemos que si $\phi_{1} \rightarrow \phi_{2}$ entonces: $S(\cdot) \phi_{1} \rightarrow S(\cdot) \phi_{2}$.

Corolario 3. Con las hipótesis del precedente teorema obtenemos:

1. Si $\phi \in H_{\text {per }}^{s}$, entonces $S(t) \phi \in H_{\text {per }}^{r} y\|S(t) \phi\|_{r} \leq\|\phi\|_{s}$, esto es, $S(t) \in \mathcal{L}\left(H_{\text {per }}^{s} ; H_{\text {per }}^{r}\right)$, $\forall t \geq 0, r \leq s$.

2. Si $\phi \in H_{p e r}^{s}$, entonces $S(\cdot) \phi \in C\left([0, \infty), H_{\text {per }}^{r}\right), \forall r \leq s$.

3. La aplicación $\phi \rightarrow S(\cdot) \phi$ es continua y verifica:

$$
\begin{aligned}
\left\|S(t) \phi_{1}-S(t) \phi_{2}\right\|_{r} & \leq\left\|\phi_{1}-\phi_{2}\right\|_{s}, \forall t \geq 0, \forall r \leq s, \\
\sup \left\|S(t) \phi_{1}-S(t) \phi_{2}\right\|_{r} & \leq\left\|\phi_{1}-\phi_{2}\right\|_{s}, \forall r \leq s .
\end{aligned}
$$

4. Si $r>s$, entonces

$$
\|S(t) \phi\|_{r} \leq \sqrt{C^{*}}\|\phi\|_{s}, \forall t>0, \forall \phi \in H_{p e r}^{s}
$$

Ahora estamos listos para enunciar y demostrar el siguiente resultado.

Teorema 4. Sea $\beta>0, s \in \mathbb{R} y\{S(t)\}_{t \geq 0}$ el semigrupo de clase $C_{0}$ del teorema 3 , entonces $S(t) \phi$ es la única solución de:

$$
(P) \mid \begin{aligned}
& u \in C\left([0, \infty), H_{p e r}^{s}\right) \\
& u_{t}=A u \in H_{p e r}^{s-4} \\
& u(0)=\phi \in H_{p e r}^{s} .
\end{aligned}
$$

En el sentido que

$$
\lim _{h \rightarrow 0}\left\|\frac{S(t+h) \phi-S(t) \phi}{h}-A S(t) \phi\right\|_{s}=0
$$

donde: $A:=-\partial_{x}^{3}-\beta\left(\partial_{x}^{4}+\partial_{x}^{2}\right)$, y si $\phi_{1} \rightarrow \phi_{2}$ entonces $S(\cdot) \phi_{1} \rightarrow S(\cdot) \phi_{2}$ además, la siguiente regularidad es satisfecha: Si $\phi \in H_{\text {per }}^{s}$, entonces $S(t) \phi \in H^{\infty}, \forall t>0$ y existe una constante $C>0$ tal que $\|S(t) \phi\|_{r} \leq C\|\phi\|_{s}, \forall t>0 \quad y \forall r \in \mathbb{R}$.

Demostración. La prueba la hacemos ordenadamente en varios pasos. 
1. Primero probaremos que $u(t)=S(t) \phi$ satisface la diferenciabilidad (8):

$$
\begin{aligned}
& \left\|\frac{S(t+h) \phi-S(t) \phi}{h}-A S(t) \phi\right\|_{s-4}^{2} \\
& =2 \pi \sum_{k=-\infty}^{+\infty}\left(1+|k|^{2}\right)^{s-4}\left|\left(\frac{S(t+h) \phi-S(t) \phi}{h}-A S(t) \phi\right)^{\wedge}\right|^{2} \\
& =2 \pi \sum_{k=-\infty}^{+\infty}\left(1+|k|^{2}\right)^{s-4} \mid \frac{e^{\left(i k^{3}-\beta\left(k^{4}-k^{2}\right)\right)(t+h)} \widehat{\phi}(k)-e^{\left(i k^{3}-\beta\left(k^{4}-k^{2}\right)\right) t} \widehat{\phi}(k)}{h} \\
& =2 \pi \sum_{k=-\infty}^{+\infty}\left(1+|k|^{2}\right)^{s-4} \mid \frac{e^{\left(i k^{3}-\beta\left(k^{4}-k^{2}\right)\right)(t+h)}-e^{\left(i k^{3}-\beta\left(k^{4}-k^{2}\right)\right) t}}{h} \\
& \quad=2 \pi \sum_{k=-\infty}^{+\infty}\left(1+|k|^{2}\right)^{s-4}|\widehat{\phi}(k)|^{2}\left|e^{\left(i k^{3}-\beta\left(k^{4}-k^{2}\right)\right) t}\right|^{2} \mid \frac{e^{\left(i k^{3}-\beta\left(k^{4}-k^{2}\right)\right) h}-1}{h} \\
& \left.h\right|^{2} \\
& =2 \pi \sum_{k=-\infty}^{+\infty} \underbrace{\left(1+k^{2}\right)^{s}|\widehat{\phi}(k)|^{2} e^{-2 \beta\left(k^{4}-k^{2}\right) t}|M(k, h)|^{2}} \\
& \left.I_{k, t, h}\right|^{2}=
\end{aligned}
$$

donde

$$
M(k, h):=\left\{\frac{e^{\left(i k^{3}-\beta\left(k^{4}-k^{2}\right)\right) h}-1}{h}-\left(i k^{3}-\beta\left(k^{4}-k^{2}\right)\right\} .\right.
$$

Usando la regla de l'Hôpital, tenemos que $M(k, h)$ tiende a cero cuando $h \rightarrow 0$. Para intercambiar los límites necesitamos acotar el término $I_{k, t, h}$ de la serie en la igualdad (10). Previamente observamos que para $h>0$, se tiene:

$$
\begin{aligned}
\frac{e^{\left(i k^{3}-\beta\left(k^{4}-k^{2}\right)\right) h}-1}{h} & =\int_{0}^{h} \frac{1}{h} \frac{\partial}{\partial s}\left(e^{\left(i k^{3}-\beta\left(k^{4}-k^{2}\right)\right) s}\right) d s \\
& =\int_{0}^{h}\left(\frac{1}{h}\left(i k^{3}-\beta\left(k^{4}-k^{2}\right)\right) e^{\left(i k^{3}-\beta\left(k^{4}-k^{2}\right)\right) s}\right) d s,
\end{aligned}
$$


tomando norma obtenemos:

$$
\begin{aligned}
\left|\frac{e^{\left(i k^{3}-\beta\left(k^{4}-k^{2}\right)\right) h}-1}{h}\right| & \\
& \left.\leq \frac{1}{h} \int_{0}^{h} \mid\left(i k^{3}-\beta\left(k^{4}-k^{2}\right)\right) e^{\left(i k^{3}-\beta\left(k^{4}-k^{2}\right)\right) s} d s\right) \mid \\
& \leq \frac{1}{h}\left|i k^{3}-\beta\left(k^{4}-k^{2}\right)\right| \int_{o}^{h} 1 d s \\
& =\frac{1}{h}\left(|k|^{3}+\beta|k|^{4}+\beta|k|^{2}\right) h \\
& =|k|^{3}+\beta\left(|k|^{4}+|k|^{2}\right), \quad h>0 .
\end{aligned}
$$

Considerando $h<0$, suficientemente pequeño, tal que $t+h>0$ con $t \neq 0$, acotamos el término $I_{k, t, h}$ de la serie (10). De (9) se tiene:

$$
\begin{aligned}
& \left|\frac{e^{\left(i k^{3}-\beta\left(k^{2}-1\right) k^{2}\right)(t+h)}-e^{\left(i k^{3}-\beta\left(k^{2}-1\right) k^{2}\right) t}}{h}-\left(i k^{3}-\beta\left(k^{4}-k^{2}\right)\right) e^{\left(i k^{3}-\beta\left(k^{2}-1\right) k^{2}\right) t}\right| \\
& \left.=\mid e^{\left(i k^{3}-\beta\left(k^{2}-1\right) k^{2}\right)(t+h)\left[\frac{1-e^{\left(i k^{3}-\beta\left(k^{2}-1\right) k^{2}\right)(-h)}}{h}\right.}-\left(i k^{3}-\beta\left(k^{4}-k^{2}\right)\right) e^{\left(i k^{3}-\beta\left(k^{2}-1\right) k^{2}\right)(-h)}\right] \mid \\
& \left.=\mid e^{\left(i k^{3}-\beta\left(k^{2}-1\right) k^{2}\right)(t+h)\left[\frac{e^{\left(i k^{3}-\beta\left(k^{2}-1\right) k^{2}\right)(-h)}-1}{-h}\right.}-\left(i k^{3}-\beta\left(k^{4}-k^{2}\right)\right) e^{\left(i k^{3}-\beta\left(k^{2}-1\right) k^{2}\right)(-h)}\right] \mid
\end{aligned}
$$

por propiedad de norma y la desigualdad triangular se tiene:

$$
\begin{aligned}
& =\left|e^{\left(i k^{3}-\beta\left(k^{2}-1\right) k^{2}\right)(t+h)}\right| \mid \frac{e^{\left(i k^{3}-\beta\left(k^{2}-1\right) k^{2}\right)(-h)}-1}{-h} \\
& -\left(i k^{3}-\beta\left(k^{4}-k^{2}\right)\right) e^{\left(i k^{3}-\beta\left(k^{2}-1\right) k^{2}\right)(-h) \mid} \\
& \leq\left|\frac{e^{\left(i k^{3}-\beta\left(k^{2}-1\right) k^{2}\right)(-h)}-1}{-h}\right|+\left|\left(i k^{3}-\beta\left(k^{4}-k^{2}\right)\right) e^{\left(i k^{3}-\beta\left(k^{2}-1\right) k^{2}\right)(-h)}\right| .
\end{aligned}
$$

De la desigualdad anterior (11) pues $-h>0$ obtenemos:

$$
\begin{aligned}
& \leq\left\{|k|^{3}+\beta\left(|k|^{4}+|k|^{2}\right)\right\}+\left\{|i k|^{3}+\beta\left(|i k|^{4}+|k|^{2}\right)\right\} \\
& =2\left\{|k|^{3}+\beta\left(|k|^{4}+|k|^{2}\right)\right\} .
\end{aligned}
$$

Usando las desigualdades (11) y 12 podemos acotar $|M(k, h)|^{2}$ como sigue:

$$
\begin{aligned}
|M(k, h)|^{2} & \leq\left\{2\left[|k|^{3}+\beta\left(|k|^{4}+|k|^{2}\right)\right]\right\}^{2} \\
& \leq\left\{C_{4}|k|^{4}\right\}^{2} \\
& \leq C_{5}\left(|k|^{2}\right)^{4} \\
& \leq C_{5}\left(1+k^{2}\right)^{4} .
\end{aligned}
$$


Además, esto es cierto en el caso $t=0$, donde solo se usa (10), luego mayoramos el $I_{k, t, h}$ término de la serie (10). Aquí usamos la estimación (13)

$$
\begin{aligned}
I_{k, t, h} & =\left(1+k^{2}\right)^{s-4}|\widehat{\phi}(k)|^{2}|M(k, h)|^{2} e^{-2 \beta\left(k^{4}-k^{2}\right) t} \\
& \leq\left(1+k^{2}\right)^{s-4}|\widehat{\phi}(k)|^{2}|M(k, h)|^{2} \\
& \leq\left(1+k^{2}\right)^{s-4}|\widehat{\phi}(k)|^{2} \mid C_{5}\left(1+k^{2}\right)^{4} \\
& =C_{5}\left(1+k^{2}\right)^{s}|\widehat{\phi}(k)|^{2}
\end{aligned}
$$

y desde que

$$
2 \pi \sum_{k=-\infty}^{+\infty}\left(1+|k|^{2}\right)^{s}|\widehat{\phi}(k)|^{2}=\|\phi\|_{s}^{2}<\infty
$$

para $\phi \in H_{\text {per }}^{s} \operatorname{los} I_{k, t, h}$ están acotados, luego usando el M-test de Weierstrass, la serie (10) converge absolutamente y uniformemente a una función continua en $h$ y es posible intercambiar los límites obteniendo (8):

$$
\left\|\frac{S(t+h) \phi-S(t) \phi}{h}-A S(t) \phi\right\|_{s-4} \rightarrow 0 \quad \text { si } \quad h \rightarrow 0
$$

que es lo que queríamos demostrar.

2. Ahora, veamos la dependencia continua respecto al dato inicial $\phi$, es decir, que si $\phi_{1} \rightarrow \phi_{2}$ entonces: $S(t) \phi_{1} \rightarrow S(t) \phi_{2}$. En efecto:

$$
\begin{aligned}
\| S(t) \phi_{1} & -S(t) \phi_{2} \|_{s}^{2} \\
& =2 \pi \sum_{k=-\infty}^{+\infty}\left(1+|k|^{2}\right)^{s}\left|\left(S(t) \phi_{1}-S(t) \phi_{2}\right)^{\wedge}(k)\right|^{2} \\
& =2 \pi \sum_{k=-\infty}^{+\infty}\left(1+|k|^{2}\right)^{s}\left|e^{\left(i k^{3}-\beta\left(k^{4}-k^{2}\right)\right) t} \widehat{\phi_{1}}(k)-e^{\left(i k^{3}-\beta\left(k^{4}-k^{2}\right)\right) t} \widehat{\phi_{2}}(k)\right|^{2} \\
& =2 \pi \sum_{k=-\infty}^{+\infty}\left(1+|k|^{2}\right)^{s}\left|e^{\left(i k^{3}-\beta\left(k^{4}-k^{2}\right)\right) t}\right|^{2}\left|\widehat{\phi_{1}}(k)-\widehat{\phi_{2}}(k)\right|^{2} \\
& \leq 2 \pi \sum_{k=-\infty}^{+\infty}\left(1+|k|^{2}\right)^{s}\left|\left(\phi_{1}-\phi_{2}\right)^{\wedge}(k)\right|^{2}=|| \phi_{1}-\phi_{2} \|_{s}^{2}
\end{aligned}
$$

luego si $\phi_{1} \rightarrow \phi_{2}$ entonces: $S(t) \phi_{1} \rightarrow S(t) \phi_{2}$.

3. Seguidamente veamos la regularidad de la solución, es decir, que si $\phi \in H_{\text {per }}^{s}$ entonces $S(t) \phi \in H^{\infty}, \forall t>0$ y existe una constante $C>0$ tal que 
$\|S(t) \phi\|_{s} \leq C\|\phi\|_{s}, \forall t, \forall r \in \mathbb{R}$. En efecto, sea $t>0$. Para $r>s$

$$
\begin{aligned}
\|S(t) \phi\|_{r}^{2} & =2 \pi \sum_{k=-\infty}^{+\infty}\left(1+|k|^{2}\right)^{r}\left|e^{\left(i k^{3}-\beta\left(k^{4}-k^{2}\right)\right) t} \widehat{\phi}(k)\right|^{2} \\
& =2 \pi \sum_{k=-\infty}^{+\infty}\left(1+|k|^{2}\right)^{s}\left|e^{\left(i k^{3}-\beta\left(k^{4}-k^{2}\right)\right) t}\right|^{2}|\widehat{\phi}(k)|^{2}\left(1+|k|^{2}\right)^{r-s} \\
& =2 \pi \sum_{k=-\infty}^{+\infty}\left(1+|k|^{2}\right)^{s}\left|e^{2 i k^{3} t} e^{-\beta\left(k^{4}-k^{2}\right) t}\right|^{2}|\widehat{\phi}(k)|^{2}\left(1+|k|^{2}\right)^{r-s} \\
& =2 \pi \sum_{k=-\infty}^{+\infty}\left(1+|k|^{2}\right)^{s}|\widehat{\phi}(k)|^{2} \underbrace{e^{-2 \beta\left(k^{4}-k^{2}\right) t}\left(1+|k|^{2}\right)^{r-s}}_{F(k, t):=}
\end{aligned}
$$

se observa que: $F(k, t)=e^{-2 \beta\left(k^{4}-k^{2}\right) t}\left(1+|k|^{2}\right)^{r-s}$ esta acotada, es decir, existe un $C^{*}$ tal que $|F(k, t)| \leq C^{*}, \forall k \in \mathbb{Z}, t>0$ y en consecuencia:

$$
\|S(t) \phi\|_{r}^{2} \leq C^{*}\|\phi\|_{s}^{2} \text { y } S(t) \phi \in H_{p e r}^{r}, \text { para, } r>s .
$$

Ahora, si $r<s$ se sabe que $H_{p e r}^{s} \subset H_{p e r}^{r}$ y como $S(t) \phi \in H_{p e r}^{s}$, entonces, $S(t) \phi \in H_{p e r}^{r}$ con $\|S(t) \phi\|_{r} \leq\|S(t) \phi\|_{s}$ y en consecuencia:

$$
S(t) \phi \in H_{p e r}^{r}, \quad \text { para, } r<s .
$$

Por lo tanto, de (14) y (15) se tiene: $S(t) \phi \in H_{p e r}^{r}, \forall r \in \mathbb{R}, t>0$, es decir, $S(t) \phi \in H^{\infty} ; \forall t>0$ y tomando $C=\max \left\{1, \sqrt{C^{*}}\right\}$ se obtiene

$$
\|S(t) \phi\|_{s} \leq C\|\phi\|_{s}, \forall t>0 ; \forall r \in \mathbb{R} .
$$

\section{Comportamiento de $u$ cuando $\beta \rightarrow 0^{+}$}

Sea $\beta>0$, la solución del problema de Cauchy $(P)$ lo denotamos por $u_{\beta}$ donde

$$
u_{\beta}(x, t)=\sum_{k=-\infty}^{\infty} e^{\left(i k^{3}-\beta\left(k^{4}-k^{2}\right)\right) t} \widehat{\phi}(k) e^{i k x},
$$

y denotamos por $\left\{u_{\beta}\right\}_{\beta>0}$ a la familia de soluciones de $(P)$. Ahora, queremos conocer y analizar el comportamiento de $u_{\beta}(x, t)$, cuando $\beta \rightarrow 0^{+}$. Usando otra vez el M-test de Weierstrass obtenemos el siguiente resultado.

Proposición 2. Se cumplen las siguientes afirmaciones:

$$
\begin{aligned}
& \text { 1. } \lim _{\beta \rightarrow 0^{+}} u_{\beta}(x, t)=\sum_{k=-\infty}^{\infty} e^{i k^{3} t} \widehat{\phi}(k) e^{i k x} \text {. } \\
& \text { 2. } \lim _{\beta \rightarrow 0^{+}}\left\|u_{\beta}(t)-g_{0}(t)\right\|_{s}=0 \quad \text { donde } \quad g_{0}(t)=\left\{e^{i k^{3} t} \widehat{\phi}(k)\right\}^{\vee} \text {. } \\
& \text { 3. } \lim _{\beta \rightarrow 0^{+}}\left\|u_{\beta}(t)\right\|_{s}=\|\phi\|_{s} \text {. }
\end{aligned}
$$

Por lo que es natural preguntarse si el límite de una familia de soluciones es una solución de un problema de valor inicial. La respuesta es afirmativa y podemos decir más: mantiene totalmente las propiedades y parcialmente la regularidad de la familia de soluciones. A continuación, estableceremos estos resultados. Empezamos con 
Teorema 5. Sea s un número real fijo y el problema

$$
(M) \mid \begin{aligned}
& u \in C\left(\mathbb{R}, H_{\text {per }}^{s}\right) \\
& \partial_{t} u+\partial_{x}^{3} u=0 \in H_{\text {per }}^{s-3} \\
& u(0)=\phi \in H_{\text {per }}^{s} .
\end{aligned}
$$

Entonces, $(M)$ está globalmente bien puesto, esto es, $\exists ! g \in C\left(\mathbb{R}, H_{\text {per }}^{s}\right)$ verificando la ecuación $(M)$, de modo que la aplicación $: \phi \rightarrow g$ que a cada dato inicial $\phi$ le asigna la solución de $(M)$, es continuo. Además, la solución satisface:

$$
g(t) \in H_{p e r}^{r}, \forall t \in \mathbb{R}, \forall r \leq s, \text { con } \quad\|g(t)\|_{r} \leq C\|\phi\|_{s}, \forall r \leq s, \forall t \in \mathbb{R} .
$$

\section{Enfoque vía Grupos}

La solución obtenida $g(t, x)$ es la serie límite del item 1 de la proposición 2 , cuando $\beta \rightarrow 0^{+}$ en la solución

$$
u_{\beta}(x, t)=\sum_{k=-\infty}^{+\infty} e^{i k^{3} t-\beta\left(k^{2}-1\right) k^{2} t} \widehat{\phi}(k) e^{i k x},
$$

y en consecuencia es afirmativa la aseveración, de que el límite de una familia de soluciones es una solución de un problema de valor inicial. Observamos también que en el teorema anterior se trabajó para todo $t \in \mathbb{R}$, por lo cual, introduciremos una familia de operadores verificando las condiciones de ser un grupo unitario de clase $C_{0}$.

Teorema 6. Sea $s \in \mathbb{R}$. La aplicación:

$$
\begin{aligned}
T: \mathbb{R} & \rightarrow \mathcal{L}\left(H_{\text {per }}^{s}\right) \\
t & \rightarrow T(t)
\end{aligned}
$$

tal que $T(t)=e^{i k^{3} t}$, esto es, la aplicación $T(t) \phi=\left\{e^{i k^{3} t} \widehat{\phi}(k)\right\}^{\vee}, \forall \phi \in H_{\text {per }}^{s}$, entonces $\{T(t)\}_{t \in \mathbb{R}}$ es un grupo unitario de clase $C_{0}$ en $H_{p e r}^{s}$. Es más, se cumplen las siguientes afirmaciones:

1. $T(\cdot) \phi \in C\left(\mathbb{R}, H_{p e r}^{s}\right)$.

2. La aplicación $\phi \rightarrow T(\cdot) \phi$ es continua y satisface:

$$
\left\|T(t) \phi_{1}-T(t) \phi_{2}\right\|_{s}=\left\|\phi_{1}-\phi_{2}\right\|_{s}
$$

y

$$
\sup _{t \in \mathbb{R}}\left\|T(t) \phi_{1}-T(t) \phi_{2}\right\|_{s}=\left\|\phi_{1}-\phi_{2}\right\|_{s}
$$

3. $T(t) \in \mathcal{L}\left(H_{\text {per }}^{s}\right)$ y $\|T(t) \phi\|_{s}=\|\phi\|_{s}, \forall \phi \in H_{\text {per }}^{s}$.

Corolario 4. Con las hipótesis del precedente teorema 6, obtenemos

1. Si $\phi \in H_{\text {per }}^{s}$ entonces $T(\cdot) \phi \in C\left(\mathbb{R}, H_{\text {per }}^{r}\right), r<s$.

2. La aplicación $\phi \rightarrow T(\cdot)$ es continua y satisface

$$
\begin{aligned}
\left\|T(t) \phi_{1}-T(t) \phi_{2}\right\|_{r} & \leq\left\|\phi_{1}-\phi_{2}\right\|_{s}, \forall t \in \mathbb{R}, \forall r<s, \\
\sup _{t \in \mathbb{R}}\left\|T(t) \phi_{1}-T(t) \phi_{2}\right\|_{r} & \leq \phi_{1}-\phi_{2} \|_{s}, \forall r<s .
\end{aligned}
$$


3. $T(t) \in \mathcal{L}\left(H_{\text {per }}^{s}, H_{\text {per }}^{r}\right)$ y $\|T(t) \phi\|_{r} \leq\|\phi\|_{s}, \forall r<s, \forall t \in \mathbb{R}, \forall \phi \in H_{\text {per }}^{s}$.

Ahora, enunciaremos la versión del teorema 5 , en función del grupo unitario $\{T(t)\}_{t \in \mathbb{R}}$, obtenido en el teorema 6 .

Teorema 7. Sea $s \in \mathbb{R} y\{T(t)\}_{t \in \mathbb{R}}$ el grupo unitario de clase $C_{0}$ en el teorema 6 . Entonces, $T(\cdot) \phi$ es la única solución de :

$$
(M) \mid \begin{aligned}
& u \in C\left(\mathbb{R}, H_{p e r}^{s}\right) \\
& u_{t}=A_{*} u \in H_{p e r}^{s-3} \\
& u(0)=\phi \in H_{p e r}^{s}
\end{aligned}
$$

en el sentido de:

$$
\lim _{h \rightarrow 0}\left\|\frac{T(t+h) \phi-T(t) \phi}{h}-A_{*} T(t) \phi\right\|_{s-3}=0
$$

donde $A_{*}:=-\partial_{x}^{3}$, y si $\phi_{1} \rightarrow \phi_{2}$ entonces $T(\cdot) \phi_{1} \rightarrow T(\cdot) \phi_{2}$. Además se tiene la siguiente regularidad: Si $\phi \in H_{\text {per }}^{s}$, entonces: $T(t) \phi \in H_{\text {per }}^{r}, \forall r \leq s, \forall t \in \mathbb{R}$ y existe una constante $C>0$ tal que $\|T(t) \phi\|_{r} \leq C\|\phi\|_{s}, \forall t \in \mathbb{R} \quad$ y $\forall r \leq s$.

\section{Conclusión}

a) La transformada de Fourier es útil para determinar, en forma intuitiva, la cara de la solución de una ecuación diferencial de tipo periódico en los espacios de Sobolev periódico dados; tal y como se hizo en la ecuación KdV-Kuramoto-Sivashinsky.

b) Fué posible dar un enfoque vía teoría de semigrupos y volver a reescribir los resultados obtenidos para la ecuación central $(P)$ haciéndola mucho mas fina.

c) Estudiamos el comportamiento en el límite vía la transformada de Fourier, de la solución $u_{\beta}$ con parámetro $\beta>0$, es decir, $\lim _{\beta \rightarrow 0^{+}} u_{\beta}$, y que este límite es también solución de un problema de valor inicial.

d) La regularidad clásica de la solución $u(t)=S(t) \phi \in C_{p e r}^{\infty}$ es consecuencia de la regularidad de dicha solución en las normas de los espacios de Sobolev y usando el Lema de inmersión de Sobolev.

e) Usamos la teoría de grupos unitarios y obtenemos resultados de existencia en el límite $\beta \rightarrow 0^{+}$de la familia de soluciones $\left\{u_{\beta}(t)\right\}_{\beta>0}$ del problema KdV-K-S.

f) Cabe mencionar que en los enfoques vía semigrupos y grupos de las dos ecuaciones diferentes diferenciales, a pesar de que los operadores diferenciales involucrados no son acotados, no se necesitó de los teoremas de existencia de Hille-Yosida (para el caso de semigrupos) o el teorema de Stone (para el caso de grupos). Los teoremas de Hille-Yosida y Stone son criterios fundamentales que proporcionan existencia de solución, pero no muestran la cara de dicha solución. Estos teoremas no se usaron debido a que se trabajó directamente con la forma que tenían dichos operadores, evidenciando la cara de la solución, gracias a la transformada inversa de Fourier. 


\section{Referencias bibliográficas}

[1] Biagioni, H., Bona, J., Iorio, R. y Scialom, M. (1996). On the Korteweg de Vries Kuramoto Sivashinsky equation. Advances in Difference Equations, 1(1), 1-20.

[2] Iorio, R. (2002). Fourier Analysis and partial Differential Equations. Cambridge, United Kingdom: Cambridge University.

[3] Kato, T. (1983). On the Cauchy problem for the (generalized) KdV equations. Advances in Mathematics Supplementary Studies, Studies in Applied Math, 8, 93-128.

[4] Lima, E. (1997). Análisis Real (Vol. 1). Lima, Perú: Colección Textos del IMCA.

[5] Liu, Z. y Zheng, S. (1999). Semigroups associated with dissipative system. New York, States United: Chapman \& Hall/CRC.

[6] Muñoz, J. (2007). Semigrupos e Equações Diferenciais Parciais. Petropolis, Brasil: LNCC.

[7] Pazy, A. (1983). Semigruops of linear Operator and applications to partial differential equations. Berlin, Alemania: Springer Verlag.

[8] Santiago, Y. y Rojas, S. (2017). Regularity and wellposedness of a problem to one parameter and its behavior at the limit. Bulletin of the Allahabad Mathematical Society, 32(1), $207-230$.

[9] Santiago, Y., Rojas, S. y Quispe, T. (2016). Espacios de Sobolev periódico y un problema de Cauchy asociado a un modelo de ondas en un fluido. Theorema, Segunda Época, 3(4), $7-23$.

[10] Topper, J. y Kawahara, T. (1978). Approximate Equations for Long Nonlinear Waves on a Viscous Fluid. Journal of the Physical Society of Japan, 44, 663-666. 